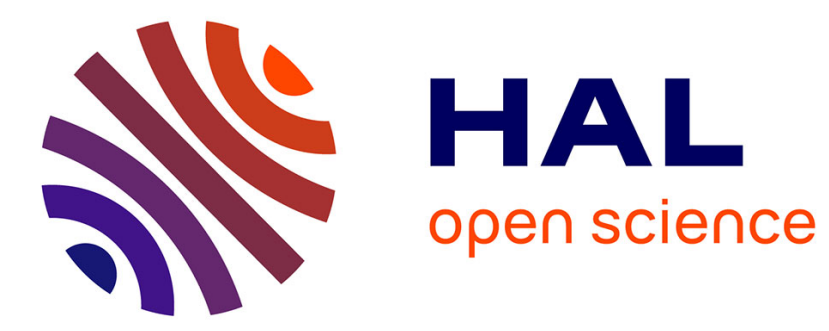

\title{
What favors convective aggregation and why?
}

Caroline Muller, Sandrine Bony

\section{To cite this version:}

Caroline Muller, Sandrine Bony. What favors convective aggregation and why?. Geophysical Research Letters, 2015, 42 (13), pp.5626-5634. 10.1002/2015GL064260 . hal-01187680

\section{HAL Id: hal-01187680 \\ https://hal-polytechnique.archives-ouvertes.fr/hal-01187680}

Submitted on 31 Aug 2015

HAL is a multi-disciplinary open access archive for the deposit and dissemination of scientific research documents, whether they are published or not. The documents may come from teaching and research institutions in France or abroad, or from public or private research centers.
L'archive ouverte pluridisciplinaire HAL, est destinée au dépôt et à la diffusion de documents scientifiques de niveau recherche, publiés ou non, émanant des établissements d'enseignement et de recherche français ou étrangers, des laboratoires publics ou privés. 


\section{RESEARCH LETTER}

10.1002/2015GL064260

\section{Key Points:}

- Longwave radiative feedbacks can

trigger a radiative aggregation

- The vertical profile of radiative cooling is crucial for self-aggregation

- Conditions favoring weak downdrafts can trigger a moisture-memory aggregation

Supporting Information:

- Figure S1

- Figure S2

- Figure S3

- Figure S4

Correspondence to:

C. Muller,

carolinemuller123@gmail.com

\section{Citation:}

Muller, C., and S. Bony (2015), What favors convective aggregation and why?, Geophys. Res. Lett., 42, 5626-5634,

doi:10.1002/2015GL064260.

Received 16 APR 2015 Accepted 3 JUN 2015

Accepted article online 5 JUN 2015 Published online 14 JUL 2015

\section{What favors convective aggregation and why?}

\author{
Caroline Muller ${ }^{1}$ and Sandrine Bony ${ }^{2}$ \\ ${ }^{1}$ CNRS/Laboratoire d'Hydrodynamique de I'École Polytechnique, Palaiseau, France, ${ }^{2}$ LMD/IPSL, CNRS, Université Pierre et \\ Marie Curie, Paris, France
}

\begin{abstract}
The organization of convection is ubiquitous, but its physical understanding remains limited. One particular type of organization is the spatial self-aggregation of convection, taking the form of cloud clusters, or tropical cyclones in the presence of rotation. We show that several physical processes can give rise to self-aggregation and highlight the key features responsible for it, using idealized simulations. Longwave radiative feedbacks yield a "radiative aggregation." In that case, sufficient spatial variability of radiative cooling rates yields a low-level circulation, which induces the upgradient energy transport and radiative-convective instability. Not only do vertically integrated radiative budgets matter but the vertical profile of cooling is also crucial. Convective aggregation is facilitated when downdrafts below clouds are weak ("moisture-memory aggregation"), and this is sufficient to trigger aggregation in the absence of longwave radiative feedbacks. These results shed some light on the sensitivity of self-aggregation to various parameters, including resolution or domain size.
\end{abstract}

\section{Introduction}

The spatial organization of deep convection is ubiquitous in the tropics, but the underlying mechanisms remain poorly understood and may not be well represented in global climate models. The spontaneous organization of convection in high-resolution models with homogeneous forcing is a useful starting point for theories of convective organization. One particular mode of convective organization that has received attention recently is the self-aggregation of convection [see, for instance, Held et al., 1993; Raymond and Zeng, 2000; Bretherton et al., 2005; Sobel et al., 2007; Muller and Held, 2012; Tobin et al., 2012; Craig and Mack, 2013; Jeevanjee and Romps, 2013; Emanuel et al., 2014]. Under certain conditions, high-resolution simulations of Radiative-Convective Equilibrium (RCE) under perfectly homogeneous forcing can exhibit a spontaneous aggregation of convection into a single region. The self-aggregated climate is a spatially organized atmosphere composed of two distinct regions, a moist region with deep clouds and intense convection and a dry subsiding environment with strong radiative cooling to space.

As shown in both simulations and observations, this phenomenon strongly impacts the large scales; in particular, aggregation is associated with drier conditions and increased outgoing longwave radiation [Bretherton et al., 2005; Tobin et al., 2012]. It has been recently suggested that the self-aggregated state could be the preferred stable equilibrium of tropical convection under warm sea surface temperatures [Emanuel et al., 2014]. With global warming, the tropics could therefore switch to this self-aggregated state. Given the large changes in large-scale properties accompanying self-aggregation, this may have important implications for climate sensitivity [Bony et al., 2015; Mauritsen and Stevens, 2015]. Self-aggregation is also believed to play a role in cyclogenesis [Khairoutdinov and Emanuel, 2013; Shi and Bretherton, 2014]. But the conditions that favor self-aggregation remain unclear.

The study of self-aggregation is complicated by the fact that the system exhibits hysteresis [Khairoutdinov and Emanuel, 2010]. Muller and Held [2012, hereafter MH12] find that when started from homogeneous initial conditions, self-aggregation is sensitive to resolution and domain size, with coarse resolutions and large domains favoring its onset. When started from aggregated initial conditions though, aggregation is maintained regardless of the resolution, as long as the domain is large enough. The hysteresis suggests that different physical mechanisms can trigger or maintain aggregation. Indeed, different initial conditions can excite different physical processes, which could exhibit different sensitivities to parameters (e.g., domain size or resolution).

In the simulations of $\mathrm{MH12}$, radiative processes, in particular in the longwave, have been shown to play a crucial role. Other feedbacks, such as those related to interactive surface fluxes or shortwave radiation, can impact 
aggregation - the former tends to favor self-aggregation, while the latter opposes it (MH12) — but neither is crucial, only longwave radiation is. There is no self-aggregation in simulations with horizontally homogeneous longwave radiative cooling rates. In particular, cloud longwave radiation plays a key role (MH12): radiation from low clouds is necessary to trigger the aggregation; once convection is aggregated though, low clouds can be made transparent to the radiation, and the aggregation persists as long as high clouds interact with radiation.

Clear-sky longwave radiation has also been identified as a key ingredient in a theoretical model of self-aggregation [Emanuel et al., 2014]. The theory predicts that above a critical specified sea surface temperature, the ordinary RCE state becomes linearly unstable to large-scale overturning circulations, due to large clear-sky infrared opacity of the lower troposphere when the free troposphere is dry.

Cold pools have been shown to impact the aggregation as well [Jeevanjee and Romps, 2013]. These authors find that in cloud-resolving simulations without cold pools, self-aggregation occurs regardless of the domain size. The domain size dependence of aggregation is therefore interpreted as resulting from cold pools interacting with the deep convection.

Here we clarify which aspects of each physical process contribute to the aggregation, using idealized experiments from a cloud-resolving model. More specifically, we address the following questions: What aspect of the low-cloud longwave radiation helps trigger the aggregation from homogeneous initial conditions? What aspect of the high-cloud longwave radiation helps maintain the aggregation? What role do cold pools play? And why is the self-aggregation favored over large domains or when coarse resolution is used?

The next section describes the numerical simulations. Section 3 investigates the role of low-cloud longwave radiation in the onset of aggregation, and section 4 addresses the role of high-cloud longwave radiation in its maintenance. Section 5 examines the role of cold pools, and conclusions are presented in section 6.

\section{Numerical Simulations and Methods}

The cloud-resolving model used in this study is the System for Atmospheric Modeling version 6.10; see Khairoutdinov and Randall [2003] for a full description. The model solves the anelastic continuity, momentum, and tracer conservation equations. The prognostic thermodynamic variables of the model include total nonprecipitating water (vapor + cloud water + cloud ice) and total precipitating water (rain + snow + graupel). We focus on radiative feedbacks and hence remove surface flux interactions by homogenizing fluxes horizontally at each time step. All simulations are three-dimensional on a square, doubly periodic horizontal domain, without rotation and with a constant and horizontally uniform sea surface temperature of $300 \mathrm{~K}$. The vertical grid has 64 levels (capped at $27 \mathrm{~km}$ with a rigid lid), with the first level at $37.5 \mathrm{~m}$ and grid spacing gradually increasing from $80 \mathrm{~m}$ near the surface to $400 \mathrm{~m}$ above $5 \mathrm{~km}$. To reduce gravity wave reflection and buildup, Newtonian damping is applied to all prognostic variables in the upper third of the model domain. The model is run to RCE, and all the outputs are shown at the end of the simulations, after 50 days of run (instantaneous snapshots at the end of the simulations are shown, unless otherwise noted).

The initial conditions are identical to $\mathrm{MH12}$, namely, to investigate the onset of aggregation, the model is initialized with horizontally homogeneous profiles from a mean tropical sounding with similar sea surface temperature, with white noise added to the dry static energy in the lowest five levels of the model to initiate the convection. To investigate the maintenance of aggregation, the runs are started from aggregated initial conditions, with the water vapor mixing ratio initialized as a "moist bubble" in the center of the domain.

In section 3, we perform simulations with fully interactive radiation, and simulations with prescribed radiative cooling profiles, imposing contrasted radiative cooling profiles in moist and dry regions. The two contrasting profiles correspond to the radiative cooling rates averaged over moist or dry regions in the runs with interactive radiation. The moist region is identified by a precipitable water threshold $\mathrm{PW}>\{80 \% \mathrm{PWmax}\}$ (profiles are robust to reasonable changes in the threshold value).

In section 4, we perform simulations with interactive radiation but assuming that low clouds are transparent to radiation. Another set of simulations is run with prescribed radiative cooling profiles in moist and dry regions. Now those two profiles correspond to regions with and without high clouds (defined as ice water path larger than $0.5 \mathrm{~g} \mathrm{~m}^{-2}$ ) in the interactive radiation runs. But for simplicity, in the simulations with prescribed radiation, we still impose them in the moist and dry regions defined by PW $>\{80 \%$ PWmax $\}$ (because regions with high 
(a) Interactive radiation:

Radiative cooling profiles averaged in dry $/$ moist regions $(\mathrm{L}=360 \mathrm{~km})$

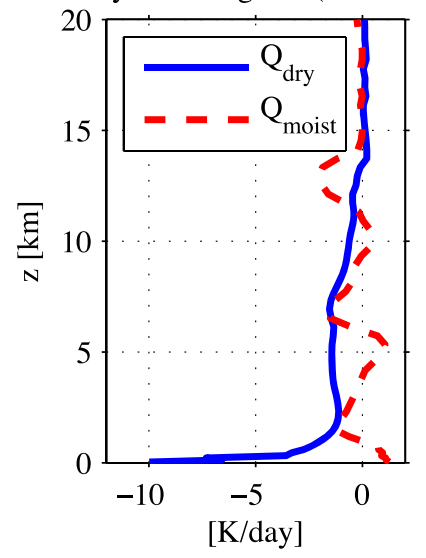

(b) $\mathrm{L}=360 \Delta \mathrm{x}=3$ (c) $\mathrm{L}=288 \Delta \mathrm{x}=3$ (d)
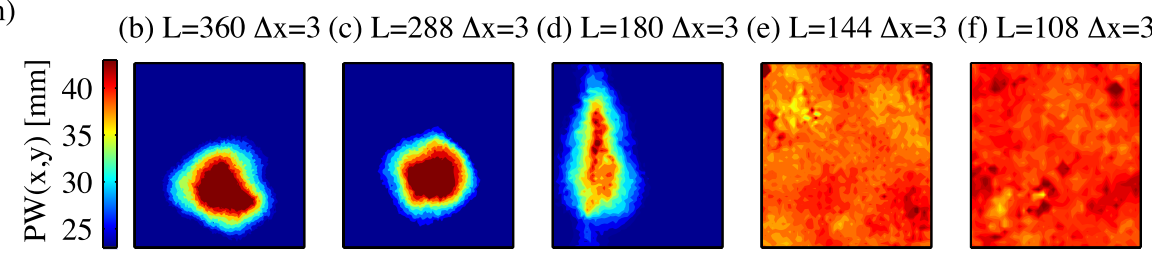

(g) $L=180 \Delta x=1$

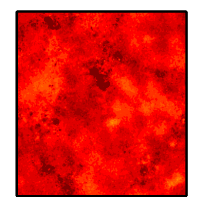

(h) Imposed radiative cooling profiles
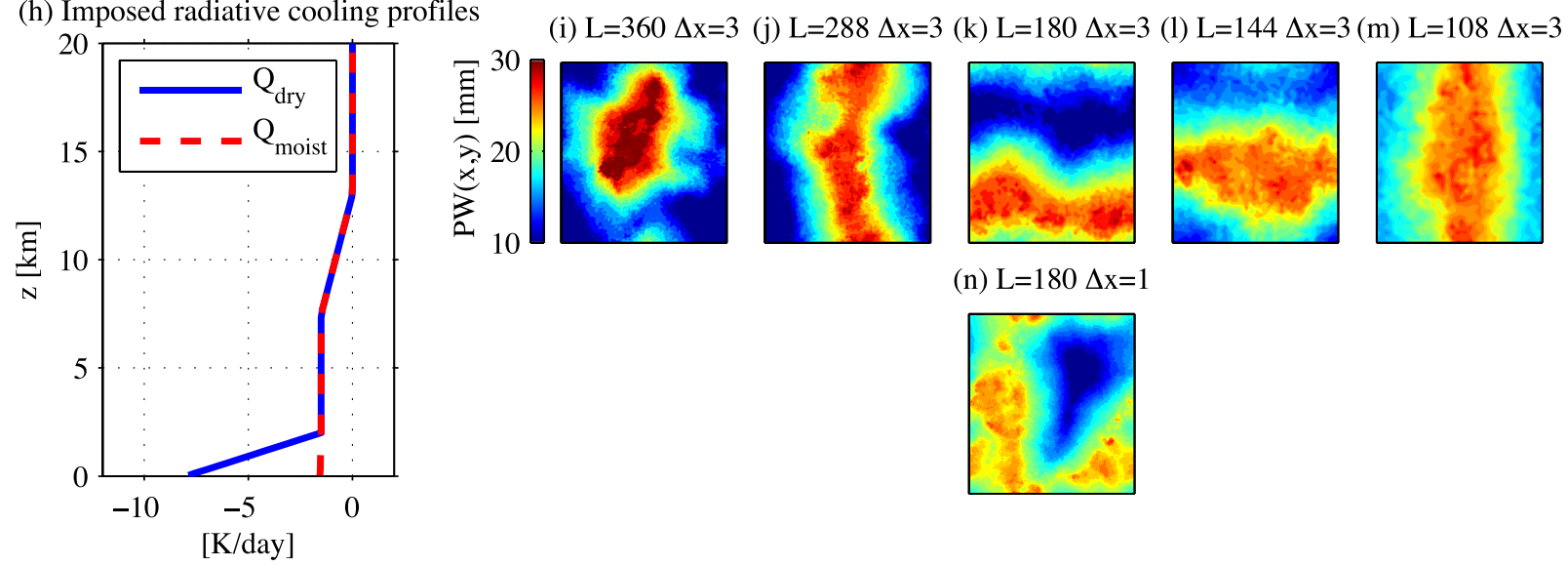

(n) $\mathrm{L}=180 \Delta \mathrm{x}=1$

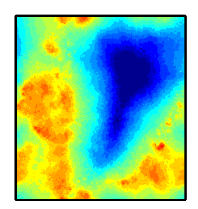

Figure 1. $(a-g)$ Simulations with interactive radiation; $(h-n)$ simulations with imposed radiative cooling profiles. Figure 1a shows the radiative cooling profile in the large domain simulation averaged in the dry (blue) and moist (red) regions shown in Figure $1 \mathrm{~b}$ (moist is defined here as precipitable water PW $>80 \%$ PWmax). In the fixed radiation runs shown in Figures $1 \mathrm{~h}-1 \mathrm{n}$, two contrasting profiles are imposed in dry and moist regions, shown as the blue and red curves in Figure $1 \mathrm{~h}$. Figures $1 \mathrm{~b}-1 \mathrm{~g}$ and $1 \mathrm{i}-1 \mathrm{n}$ show snapshots of $\mathrm{PW}(\mathrm{mm})$ at various domain sizes $L$ and resolutions $\Delta x$ (in $\mathrm{km}$ ).

clouds closely correspond to moist convective regions). As we will see, this is sufficient to identify the features of the radiative cooling profiles which are responsible for the aggregation.

Finally, in section 5, we perform simulations with weakened downdrafts and associated cold pools. Following Jeevanjee and Romps [2013], this is done by suppressing the evaporation of rain in the lowest kilometer of the domain. These runs have prescribed, horizontally homogeneous radiative cooling rates, and interactive surface fluxes. However, the surface wind velocity used in the computation of surface turbulent fluxes is set to a constant value of $5 \mathrm{~m} \mathrm{~s}^{-1}$ to eliminate the Wind-Induced Surface Heat Exchange instability mechanism.

\section{Onset of Convection}

Here we investigate the onset of aggregation, i.e., the spontaneous aggregation of convection from homogeneous initial conditions. When started from homogeneous conditions, it has been shown that convection aggregates on large domains [Bretherton et al., 2005] and at coarse resolutions (MH12). This is consistent with Figures $1 \mathrm{~b}-1 \mathrm{~g}$, which show simulations with interactive radiation run for various domain sizes and resolutions.

The sensitivity study of $\mathrm{MH} 12$ shows that the interaction between the radiation scheme and the convection is crucial for the aggregation. Simulations with a horizontally homogeneous radiative cooling profile do not aggregate, whatever the resolution or domain size. MH12 further find that the spatial inhomogeneity of the radiative cooling due to clouds is necessary for aggregation to occur. Here we address the following question: 
is the difference in radiative cooling profiles between the convecting moist region and the dry environment responsible for the aggregation?

Figure 1 a shows the radiative cooling profiles averaged inside and outside the moist convecting region in a run with interactive radiation which self-aggregates (domain size $L=360 \mathrm{~km}$, horizontal resolution $\Delta x=3 \mathrm{~km}$ ). In the dry region, the radiative profiles exhibit a strong low-level cooling due to low-level clouds and dry air. And in the moist region, the presence of deep clouds induces a warming below and cooling above the three cloud layers (high clouds around $12 \mathrm{~km}$, middle clouds around $6 \mathrm{~km}$, and low clouds around $1 \mathrm{~km}$ ).

We perform a new simulation with imposed radiative cooling profiles but imposing the two contrasting profiles of Figure 1a inside and outside the moist region (in other words, the spatial distribution of radiative cooling rates is determined solely by the spatial distribution of PW). In that case, we find that convection aggregates for each domain size and resolution. In fact, it is sufficient to include the low-level cooling in dry regions to obtain aggregation in all the runs (Figures $1 \mathrm{~h}-1 \mathrm{n}$ ). On the other hand, imposing the radiative profile of the moist region only does not yield aggregation (supporting information Figure S1). Furthermore, imposing the low-level cooling everywhere (radiative cooling everywhere equal to $Q_{\text {dry }}$ from Figure $1 \mathrm{~h}$ ) does not yield aggregation either (not shown), consistent with the known result that convection does not aggregate with horizontally homogeneous cooling profiles. The low-level cooling in dry regions results from the strong radiative cooling of low clouds, as well as clear-sky cooling in the first kilometer or so, due to warm near-surface conditions overlaid by a dry free troposphere (Figure 2a).

Diagnostics based on the vertically integrated Moist Static Energy ( $\int$ MSE) budget [Bretherton et al., 2005; $\mathrm{MH12}$ ] and $\int \mathrm{MSE}$ variance budget [Wing and Emanuel, 2014] have been used to investigate self-aggregation and the feedbacks responsible for it. Indeed, self-aggregation is associated with a large variability of precipitable water, hence a large variability of $\int \mathrm{MSE}$, with very low values in the dry subsidence region, and high values in the moist convecting region. One measure of the time evolution of self-aggregation is therefore $\int$ MSE variability, and the various terms of the time evolution equation for the $\int$ MSE variance quantify the feedbacks contributing to aggregation, as nicely discussed in Wing and Emanuel [2014]. In those budgets, only the vertically integrated radiative cooling enters. One interesting question is whether the vertical structure of the radiative cooling also matters, or if it is the net vertically integrated value which is key for aggregation.

To address this question, we apply the same vertically integrated cooling in the dry region as in Figure $1 \mathrm{~h}$ but with a different vertical structure (constant, or with a maximum cooling at $z=2 \mathrm{~km}$ instead of at the surface). In that case, convection does not aggregate (supporting information Figure S1). Therefore, the vertical distribution of radiative cooling matters for the triggering of aggregation.

The importance of the low-level radiative cooling is consistent with earlier studies, which highlight its role in generating a low-level circulation from dry (low $\int \mathrm{MSE}$ ) to moist (high $\int \mathrm{MSE}$ ) regions associated with aggregation. The stream function in height and vertically integrated $\int$ MSE coordinates in an interactive radiation simulation which self-aggregates is shown in Figures 2a and 2b (see Bretherton et al. [2005], for details on how to compute this stream function).

The triggering of self-aggregation is associated with the development of a low-level circulation, represented schematically as arrows in Figure 2. This low-level circulation transports low-level high MSE air into the high-energy region (solid black arrow, transporting MSE around $330 \mathrm{~K}$ ), and midlevel low MSE air into the low-energy region (dashed black arrow, transporting MSE around $320 \mathrm{~K}$ ). This results in a net reduction of MSE in the low-energy region, and a net increase of MSE in the high-energy region, thereby enhancing the $\int$ MSE gradient. This upgradient MSE transport of the low-level circulation is believed to be a key positive feedback in the aggregation process [Bretherton et al., 2005]. Not only does the strength of the circulation matter, but its height as well, since the MSE of the flow is largely determined by its height (Figure $2 \mathrm{~b}$ ). Both the strength and height are influenced by radiation, since low-level cooling in dry regions promotes subsidence (blue arrow in Figures $2 \mathrm{a}$ and $2 \mathrm{~b}$ ), which in turn forces a low-level return flow from dry to moist areas (solid black arrow).

It is therefore the spatial variability of the low-level cooling which yields aggregation. This may explain the sensitivity to domain size and resolution. Indeed, Khairoutdinov et al. [2009] show that low-level cloud amounts increase at coarse resolution. In our simulations we also find that the condensate amount in clouds is larger at coarser resolution, as well as on larger domains (supporting information Figure S2). This may lead to larger contrasts in radiative cooling rates between regions with/without low-level clouds, and thus a larger 
(a) Interactive radiation

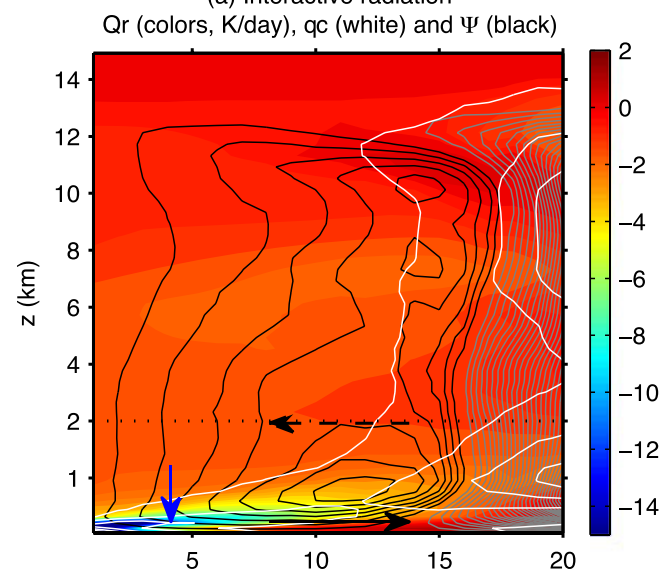

(c) Interactive radiation without low clouds Qr (colors, K/day), qc (white) and $\Psi$ (black)

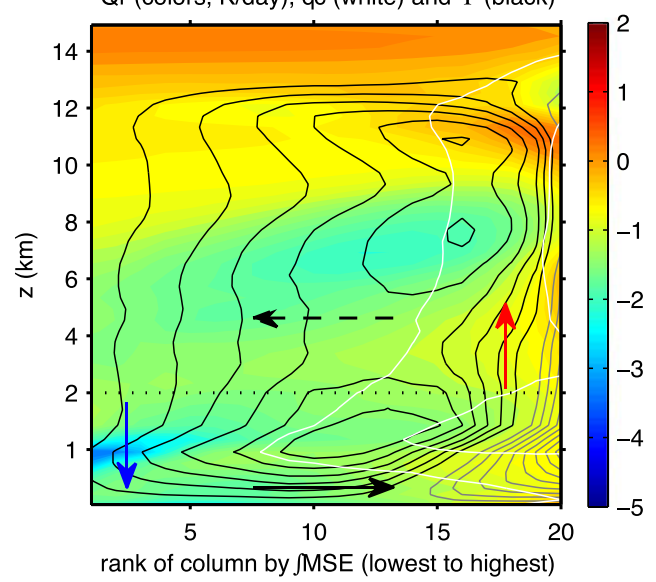

(b) Same as panel a but the colors show MSE (K)

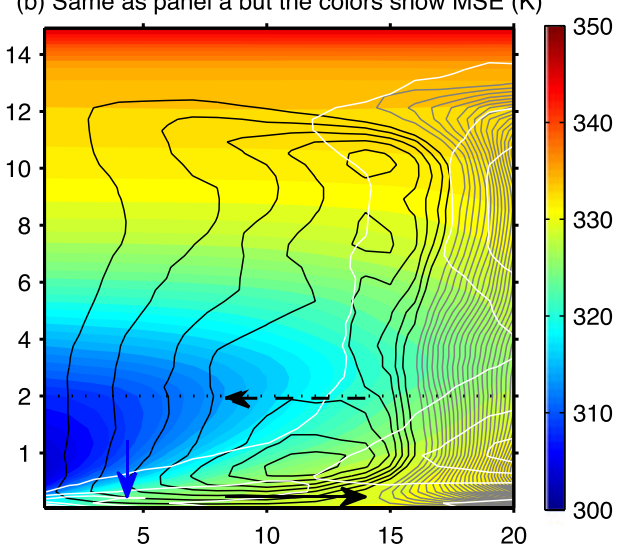

(d) Same as panel c but the colors show MSE (K)

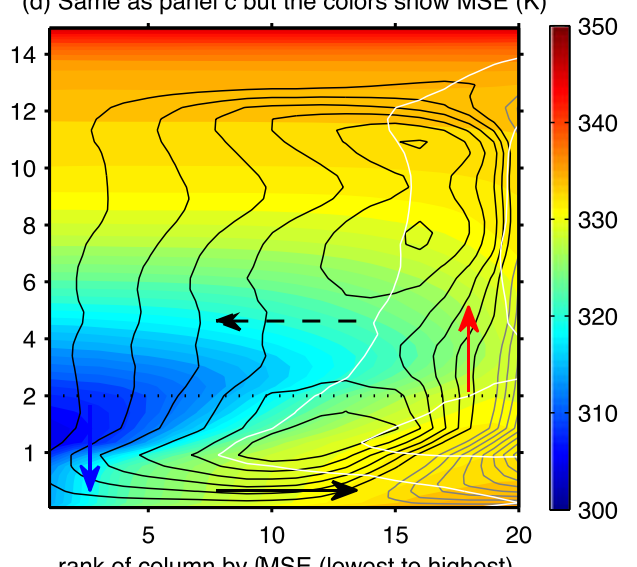

Figure 2. Radiative cooling rates (colors in Figures $2 \mathrm{a}$ and $2 \mathrm{c}$ ), moist static energy (colors in Figures $2 \mathrm{~b}$ and $2 \mathrm{~d}$ ), cloud water content (liquid + ice, white contours every $5 \times 10^{-2} \mathrm{~g} \mathrm{~kg}^{-1}$ starting at $5 \times 10^{-3} \mathrm{~g} \mathrm{~kg}^{-1}$ ), and stream function (black contours for counterclockwise, gray contours otherwise, every $8 \times 10^{-3} \mathrm{~kg} \mathrm{~m}^{-2} \mathrm{~s}^{-1}$ ), averaged over the last 20 days of aggregated simulations, plotted as a function of height $z$ and vertically integrated moist static energy $\int$ MSE. Note the stretched vertical coordinate $z$ below $2 \mathrm{~km}$. (a, b) Simulations with fully interactive radiation; $(c, d)$ similar simulation but without the low-cloud radiative effects. The arrows schematically represent the subsidence generated by the radiative cooling (blue) and rising motion by the warming (red), as well as the low-level (solid black) and midlevel (dashed black) flows induced.

spatial variability of radiative cooling rates with both coarser resolutions and larger domains, which favors self-aggregation.

\section{Maintenance of Convection}

Here we investigate the maintenance of aggregation, i.e., the evolution of simulations which started from aggregated initial conditions. Although the radiative cooling from low-level clouds is crucial for the onset of aggregation, it is not necessary for its maintenance (MH12). Aggregated runs remain aggregated even without radiative cooling from low clouds, as long as high clouds interact with the radiation and the domain size is large enough. In that case, no sensitivity to resolution is observed (MH12).

Simulations started from aggregated initial conditions with interactive radiation, but removing contributions from low clouds are shown on the top panels of Figure 3 . The radiative contribution from low clouds is removed by setting the liquid condensate amount that enters the radiation computation to zero (liquid water clouds occur only within the first few kilometers above the surface). In other words, only ice clouds impact radiative cooling rates. Since resolution does not play a role in the maintenance of aggregation, we only investigate the sensitivity to domain size and use the same resolution $(3 \mathrm{~km})$ in all the runs. As expected, the 
(a) Interactive radiation without low clouds: Radiative cooling profiles averaged in clear/cloudy regions $(\mathrm{L}=720 \mathrm{~km})$

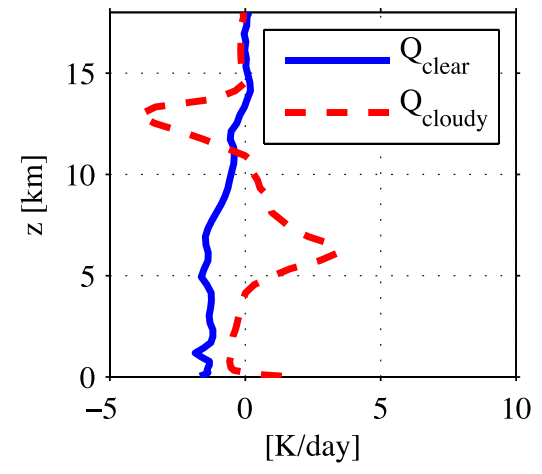

(b) $\mathrm{L}=720$

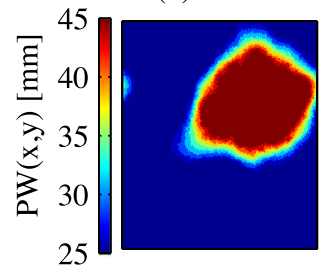

(c) $\mathrm{L}=540$

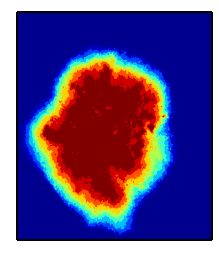

(d) $\mathrm{L}=360$

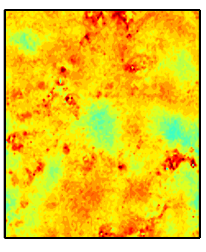

(e) $\mathrm{L}=180$

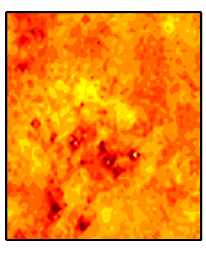

(f) Imposed radiative cooling profiles

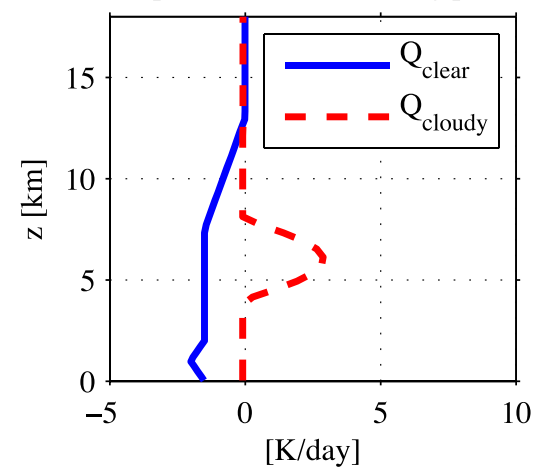

(g) $\mathrm{L}=720$

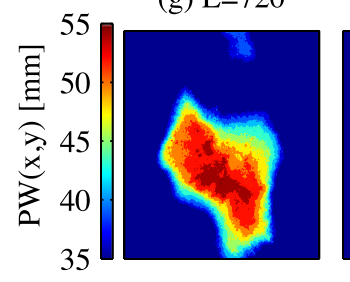

(h) $\mathrm{L}=540$

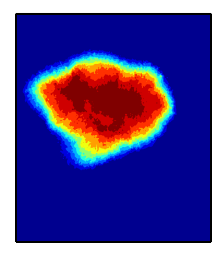

(i) $\mathrm{L}=360$

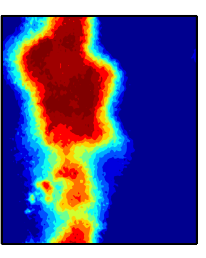

(j) $\mathrm{L}=180$

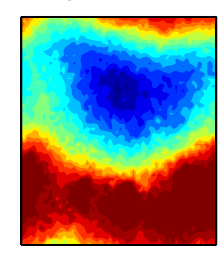

Figure 3. Simulations started from aggregated initial conditions. (a-e) Simulations with interactive radiation except radiation from low-level clouds. Low-cloud radiation is turned off by setting to zero the liquid cloud amount entering the radiation computation. Figure 3a shows the radiative cooling profile of the large domain simulation averaged in the clear (blue) and cloudy (red) region (cloudy is defined here as ice water path greater than $0.5 \mathrm{~g} \mathrm{~m} \mathrm{~m}^{-2}$ ); Figures $3 \mathrm{~b}-3 \mathrm{e}$ show precipitable water PW $(\mathrm{mm})$ at various domain sizes (and with same resolution $3 \mathrm{~km})$. ( $(\mathrm{f}-\mathrm{j})$ Simulations with imposed radiative cooling profiles. Two different profiles are imposed in the moist (red) and dry (blue) regions (Figure $3 f$ ); Figures $3 g-3 j$ show precipitable water PW (mm).

initial aggregation is only maintained on large domains. The radiative profiles inside and outside the cloudy region (Figure 3a) differ from runs with low-cloud radiation, in particular the low-level clear-sky cooling outside the convective region is weaker and occurs at a higher altitude (minimum of $-2 \mathrm{~K} \mathrm{~d}^{-1}$ around $1 \mathrm{~km}$ above the surface. This peak in cooling results from the strong clear-sky cooling of low levels induced by the dry free troposphere and the warm near-surface temperatures, which increases the longwave cooling from low levels).

As in section 3, we investigate whether imposing the contrasting radiative cooling rates inside and outside the moist convecting region maintains aggregation. When these two profiles are imposed, we find that aggregation is maintained for all domain sizes. In fact, it is sufficient to include the low-level clear-sky cooling of the dry regions and the midlevel warming of the moist regions to maintain aggregation for all domain sizes (Figures $3 f-3 j$ ). Interestingly, in that case, imposing either of these separately does not yield aggregation at all domain sizes (supporting information Figure S3). We note also that the upper level part (10-15 km) or the low part (below $4 \mathrm{~km}$ ) of the cloudy profile can contribute to maintain aggregation, though only the middle part yields aggregation at all domain sizes (supporting information Figure S4; to ease comparison, a constant cooling is added to the profiles to maintain the same vertically integrated net cooling). This again implies that not only does the vertically integrated radiative cooling matter but the vertical profile of radiative cooling is important as well. Changing the vertical structure of the cooling can make the convection disaggregate.

The low part of the radiative profile in the dry region is particularly critical, owing to its role in the low-level circulation discussed in the previous section, but it is weaker and is not sufficient by itself to maintain the aggregation (Figure $2 \mathrm{c}$ ). To maintain the aggregation, the low-level cooling in the dry region must be accompanied by midlevel radiative warming in the moist region caused by clouds. It again has to do with the 


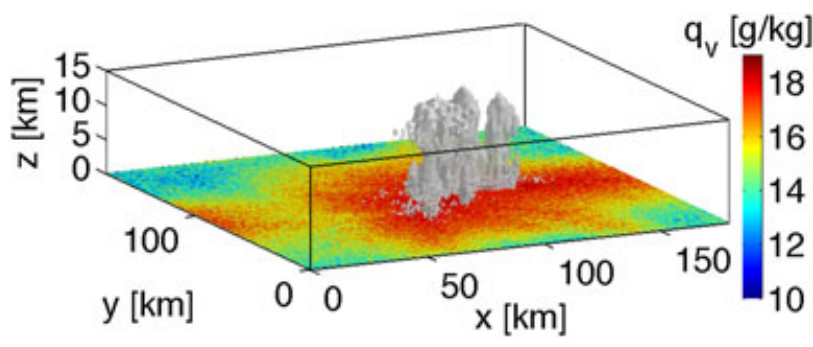

Figure 4. Clouds (white surfaces) and near-surface water vapor mixing ratio (first atmospheric level at $37.5 \mathrm{~m}$ in colors) at the end of a simulation without cold pools. The cold pools and associated downdrafts have been removed by suppressing the reevaporation of rain in the first kilometer as in Jeevanjee and Romps [2013]. The radiative cooling profile is imposed and the same at every location, equal to $-1.5 \mathrm{~K} \mathrm{~d}^{-1}$ in the troposphere everywhere in the domain (linearly decreasing to zero in the stratosphere, dashed red profile in Figure $1 \mathrm{~h})$. low-level dynamics (schematically represented by arrows in Figures $2 c$ and $2 d$ ) and the associated MSE transport. The low-level cooling generates subsidence (blue arrow), and the midlevel warming enhances the upward motion in the moist region (red arrow), generating a low-level circulation (black arrows). This low-level circulation transports low-level high MSE air into the high-energy region (solid black arrow), and midlevel low MSE air into the low-energy region (dashed black arrow), thereby enhancing the $\int$ MSE gradient. As in the onset of aggregation, both the strength and the height of the radiative cooling/warming matter, since they determine the strength and height of the

low-level circulation and hence the associated MSE transport. Not only are high-cloud radiative effects important for the maintenance of aggregation, but the clear-sky cooling in the subcloud layer also plays a key role.

\section{Role of Cold Pools}

It has recently been suggested that cold pools could play an important role in the dependence of aggregation on domain size. Jeevanjee and Romps [2013] show that simulations started from aggregated initial conditions remain aggregated at all domain sizes when the cold pools are weakened.

We also find that weakening cold pools yields aggregation at all domain sizes. But the simulations aggregate even if we impose a fixed horizontally homogeneous radiative cooling profile $\left(-1.5 \mathrm{~K} \mathrm{~d}^{-1}\right.$ in the troposphere everywhere in the domain, decreasing to zero in the stratosphere, dashed red profile in Figure $1 \mathrm{~h}$ ). This implies that the feedback involved is different from the longwave radiative feedback responsible for aggregation in other studies. Instead, convection remains aggregated at the same location throughout the run because of the absence of evaporation-driven downdrafts below deep convective clouds.

In standard conditions, the cooling associated with the evaporation of rain below deep convecting clouds generates downdrafts, which through their thermodynamical effect oppose the upward motion that generated the cloud. This negative feedback on upward convection suppresses the deep cloud in a few hours. Convection tends to occur in moist areas. Without the evaporation of rain and the effect of the associated downdrafts, moist areas remain moist (or even get moister by convergence) and thus become even more favorable to convection. This tends to "localize" the convection, as observed in our simulations (Figure 4). As mentioned in section 2, in those simulations, feedbacks associated with interactions between surface winds and surface fluxes have been removed. We expect that they would make the aggregation even more vigorous (enhanced moisture fluxes by surface convergent winds). The feedback responsible for the aggregation in that case in not a radiative feedback, but a "moisture-memory" feedback [Tompkins, 2001]. This moisture-memory self-aggregation of convection is relevant in situations where downdrafts are weak, as is the case for instance when the subcloud layer is nearly saturated (as, for instance, in hurricanes). This is reminiscent of the theoretical model for aggregation proposed by Craig and Mack [2013], which accounts for moisture-memory feedbacks but not radiative effects.

\section{Conclusions}

We find that several physical processes can give rise to self-aggregation. Longwave radiative feedbacks yield a "radiative aggregation." More precisely, the contrasting radiative cooling rates inside and outside the moist convecting region generate a low-level circulation, which transports MSE upgradient and helps trigger or maintain the aggregation process [Bretherton et al., 2005]. We show that this may explain why self-aggregation is favored under certain conditions, such as large domains and coarse resolutions: the contrast in cooling rates, and hence the strength of the circulation that results from it, may increase with larger variability on 
larger domains and at coarse resolutions. This is consistent with larger cloud condensate amounts on larger domains and at coarser resolutions [Khairoutdinov et al., 2009], leading to larger spatial variability of radiative cooling rates between regions with clouds and regions without. Artificially imposing the radiative variability yields aggregation at all domain sizes and resolutions.

Conditions in which downdrafts below clouds are weak can give rise to a moisture-memory aggregation. This behavior results from the absence of low-entropy advection into the subcloud layer by evaporative-driven downdrafts below clouds, which would otherwise kill convective cells within a few hours. As a consequence, convection remains at the same humid location throughout the simulation. In that case, convection remains aggregated even if radiative feedbacks are turned off. The simple model proposed by Craig and Mack [2013], which includes moisture effects, may very well capture this coarsening feedback. This feedback may be relevant in cases where the subcloud layer is nearly saturated.

The variety of processes leading to aggregation may explain its presence in different model runs in various configurations [e.g., Bretherton et al., 2005; Muller and Held, 2012; Jeevanjee and Romps, 2013; Shi and Bretherton, 2014; Wing and Emanuel, 2014] and its hysteresis, since evolving conditions can trigger various feedbacks. Conditions leading to a large radiative variability can more easily trigger radiative aggregation, while a humid subcloud layer can more easily yield moisture-memory convective coarsening.

We find that not only do vertically integrated radiative budgets matter for aggregation, but the vertical profile of cooling is also key. The vertically integrated radiative terms typically yield positive feedbacks on MSE gradients, mainly due to reduced longwave cooling in moist regions and enhanced cooling in dry regions. This is true whether or not convection is aggregated (MH12). What is specific to aggregation is the fact that the circulation does not oppose the energy gradient by transporting MSE from the moist to the dry region. Instead, the circulation reinforces the MSE gradient by transporting energy from the dry to the moist convecting region. This work suggests that the vertical distribution of radiative cooling in the dry and moist regions is key to the dynamics and associated MSE transport. This is because MSE is a strong function of height, with high MSE near the surface and lower MSE values at midlevels. The vertical profile of radiation dictates the height of the dynamic response, which in turn dictates the MSE transport between dry and moist regions.

Self-aggregation has been shown to be sensitive to temperature, being favored not only at warm but also at cold temperatures [Khairoutdinov and Emanuel, 2010; Abbot, 2014]. Our simulations were all performed with a fixed sea surface temperature of $300 \mathrm{~K}$. The relative efficiency of these different processes may change as temperature rises. More work is needed to investigate which feedback, if any, dominates in current conditions and under different climates. High-resolution simulations under more realistic conditions, as well as a careful comparison with observations of convective aggregation in the tropics and of its impact on large scales [Tobin et al., 2012, 2013], could be useful to better understand this phenomenon and see whether the processes highlighted in this idealized study can also be found in nature.

Acknowledgments

All the data used in this study are available upon request from the authors. C.M. gratefully acknowledges financial support from the Chair for sustainable development at Ecole Polytechnique. S.B. acknowledges support from the Labex L-IPSL. We acknowledge CINES/GENCI, France, for providing access and support to their computing platform JADE.

The Editor thanks two anonymous reviewers for their assistance in evaluating this paper.

\section{References}

Abbot, D. S. (2014), Resolved snowball Earth clouds, J. Clim., 27, 4391-4402.

Bony, S., et al. (2015), Clouds, circulation and climate sensitivity, Nat. Geosci., 8, 261-268.

Bretherton, C. S., P. N. Blossey, and M. Khairoutdinov (2005), An energy-balance analysis of deep convective self-aggregation above uniform SST, J. Atmos. Sci., 62(12), 4273-4292.

Craig, G. C., and J. M. Mack (2013), A coarsening model for self-organization of tropical convection, J. Geophys. Res. Atmos., 118(16), $8761-8769$, doi:10.1002/jgrd.50674.

Emanuel, K. A., A. A. Wing, and E. M. Vincent (2014), Radiative-convective instability, J. Adv. Model. Earth Syst., 6(1), 75-90.

Held, I. M., R. S. Hemler, and V. Ramaswamy (1993), Radiative-convective equilibrium with explicit two-dimensional moist convection, J. Atmos. Sci., 50(23), 3909-3909.

Jeevanjee, N., and D. M. Romps (2013), Convective self-aggregation, cold pools, and domain size, Geophys. Res. Lett., 40, 994-998, doi:10.1002/grl.50204.

Khairoutdinov, M., and K. Emanuel (2013), Rotating radiative-convective equilibrium simulated by a cloud-resolving model, J. Adv. Model. Earth Syst., 5(4), 816-825.

Khairoutdinov, M. F., and K. A. Emanuel (2010), Aggregated convection and the regulation of tropical climate, paper presented at 29th conference on Hurricanes and Tropical Meteorology, Am. Meteorol. Soc., Tucson, Ariz., 13 May.

Khairoutdinov, M. F., and D. A. Randall (2003), Cloud-resolving modeling of the arm summer 1997 IOP: Model formulation, results, uncertainties and sensitivities, J. Atmos. Sci., 60, 607-625.

Khairoutdinov, M. F., S. K. Krueger, C. H. Moeng, P. Bogenschutz, and D. A. Randall (2009), Large-eddy simulation of maritime deep tropical convection, J. Adv. Model. Earth Syst., 1, 15, doi:10.3894/JAMES.2009.1.15.

Mauritsen, T., and B. Stevens (2015), Missing iris-effect as a possible cause of high climate sensitivity in models, Nat. Geosci., 8, 346-351 doi:10.1038/ngeo2414.

Muller, C. J., and I. M. Held (2012), Detailed investigation of the self-aggregation of convection in cloud-resolving simulations, J. Atmos. Sci., $69,2551-2565$ 
Raymond, D. J., and X. Zeng (2000), Instability and large-scale circulations in a two-column model of the tropical troposphere, Q. J. $R$. Meteorol. Soc., 126(570), 3117-3135.

Shi, X., and C. S. Bretherton (2014), Large-scale character of an atmosphere in rotating radiative-convective equilibrium, J. Adv. Model. Earth Syst., 6(3), 616-629, doi:10.1002/2014MS000342.

Sobel, A. H., G. Bellon, and J. Bacmeister (2007), Multiple equilibria in a single-column model of the tropical atmosphere, Geophys. Res. Lett., 34, L22804, doi:10.1029/2007GL031320.

Tobin, I., S. Bony, and R. Roca (2012), Observational evidence for relationships between the degree of aggregation of deep convection, water vapor, surface fluxes, and radiation, J. Clim., 25, 6885-6904.

Tobin, I., S. Bony, C. E. Holloway, J. Y. Grandpeix, G. Sèze, D. Coppin, S. J. Woolnough, and R. Roca (2013), Does convective aggregation need to be represented in cumulus parameterizations?, J. Adv. Model. Earth Syst., 5, 692-703.

Tompkins, A. M. (2001), Organization of tropical convection in low vertical wind shears: The role of water vapor, J. Atmos. Sci., 58, 529-545. Wing, A. A., and K. A. Emanuel (2014), Physical mechanisms controlling self-aggregation of convection in idealized numerical modeling simulations, J. Adv. Model. Earth. Syst., 6(1), 59-74. 